\title{
Avant-propos
}

\section{Béatrice Lovis}

\section{(2) OpenEdition}

\section{Journals}

Édition électronique

URL : https://journals.openedition.org/edl/3520

DOI : 10.4000/edl.3520

ISSN : 2296-5084

Éditeur

Université de Lausanne

\section{Édition imprimée}

Date de publication : 15 mai 2021

Pagination : 7-14

ISBN : 978-2-940331-76-5

ISSN : 0014-2026

Référence électronique

Béatrice Lovis, "Avant-propos », Études de lettres [En ligne], 315 | 2021, mis en ligne le 15 mai 2021, consulté le 17 mai 2022. URL : http://journals.openedition.org/edl/3520 ; DOI : https://doi.org/ 10.4000/edl.3520 


\section{AVANT-PROPOS}

L'histoire du théâtre a souvent eu tendance à se confondre avec celle des théâtres institutionnels, soutenus par les pouvoirs publics et animés par des troupes professionnelles. D'autre part, les littéraires se sont longtemps intéressés presque exclusivement au répertoire imprimé qui a été, de préférence, consacré sur une scène prestigieuse d'une capitale. Les conséquences d'une telle approche sont multiples. Ainsi, non seulement la vie théâtrale des régions périphériques est oubliée, mais l'intense activité des comédiens amateurs disparaît aussi dans la foulée, de même que les pièces restées à l'état de manuscrit, dont bon nombre sont de la plume de femmes, maintenues dans l'ombre en raison de leur sexe ${ }^{1}$.

Or, cette manière d'écrire l'histoire a profondément impacté l'étude du théâtre en Suisse, romande en particulier, considéré comme provincial, sans rayonnement notoire, longtemps resté sans répertoire propre, ne comptant que quelques rares acteurs professionnels et dramaturges. Souffrant d'un manque de soutien politique jusqu'au début du XX $\mathrm{X}^{\mathrm{e}}$ siècle, les arts du spectacle ont, de plus, traîné pendant plusieurs siècles une réputation sulfureuse, tout spécialement dans les cantons

I. Depuis une vingtaine d'années en France, la recherche se décentralise enfin et s'intéresse à la vie théâtrale en province, dans le sillage de Max Fuchs (cf. projet ANR Thérepsicore "Le théâtre sous la Révolution et l'Empire en province", dirigé par Philippe Bourdin). Parallèlement, on constate un intérêt marqué pour le théâtre amateur aux XVIII ${ }^{\mathrm{e}}$ et XIX ${ }^{\mathrm{e}}$ siècles, le théâtre d'éducation et celui donné sur des scènes secondaires, à l'exemple des théâtres de la Foire et des boulevards. Plusieurs études et anthologies portent aussi sur les dramaturges femmes sous l'Ancien Régime et au XIX ${ }^{\mathrm{e}}$ siècle. Voir notamment les travaux de Marie-Emmanuelle Plagnol-Diéval, Valentina Ponzetto, Françoise Rubellin, Jean-Claude Yon, Perry Gethner, Aurore Evain et d'Odile Krakovitch. 
protestants: oisiveté, luxe, immoralité, incitation à la débauche et à l'indiscipline; en d'autres termes, un divertissement nuisible, étranger aux valeurs helvétiques, largement tributaire des nations voisines. Telle a été du moins la vision caricaturale dont ont hérité les historiens du $\mathrm{XX}^{\mathrm{e}}$ siècle.

Dès la première moitié du XIX ${ }^{\mathrm{e}}$ siècle, à l'heure où le peuple suisse cherche à se constituer une identité et à se trouver un "génie national", la question du théâtre a en effet été une "épine dans le pied des historiens de la littérature suisse romande " ${ }^{2}$, comme le relève Daniel Maggetti dans son Invention de la littérature romande. Les propos du Genevois Rodolphe Töpffer sont très explicites à cet égard:

nous sommes en cette grande question [du théâtre] l'obscur adepte de notre illustre concitoyen [Rousseau] ; mais surtout, enfant comme lui d'une république qui n'a vécu et qui ne vivra que par sa foi et par ses mœurs, nous avons trop bien vu s'accomplir de notre temps, sous la délétère influence d'un théâtre étranger aujourd'hui entièrement acclimaté dans nos murs, tous les funestes résultats qu’avait prédit ce fier et vigilant républicain, pour que, appliquée à notre pays, cette question ne soit pas à nos yeux pleinement, péremptoirement résolue. Oui, malheur aux petits peuples qui, n'ayant pas, ne pouvant pas avoir une scène nationale, empruntent à de puissants voisins leurs histrions et leur théâtre, et importent au milieu d'eux, avec sympathies, de préventions qui ne leur appartiennent pas en propre, et qui devaient leur demeurer à jamais étrangers! ${ }^{3}$

À sa suite, les historiens ne feront qu'accentuer cette volonté de se distancier du théâtre français joué sur les scènes romandes au cours du XVIII ${ }^{e}$ siècle, un théâtre qui est incarné par la personnalité de Voltaire, fer de lance auquel s'est opposé Rousseau avec véhémence dans sa Lettre à D’Alembert sur les spectacles en 1758. Voltaire, écrit André Gindroz en 1853, "pendant son séjour [lausannois], depuis 1756 à 1758, produisit un mouvement un peu littéraire et très frivole. Une société brillante, légère, avide de plaisirs, apparut au sein de notre paisible cité, et Lausanne devint une ville d'amusements, de théâtre, de mœurs parisiennes et d'incrédulité voltairienne " ${ }^{4}$. Dans son Histoire littéraire

2. D. Maggetti, L'invention de la littérature romande, 1830-1910, p. 388.

3. R. Töpffer, Voyage autour du Mont-Blanc, p. 251 sq.

4. A. Gindroz, Histoire de l'instruction publique dans le Pays de Vaud, p. 136 sq. 
de la Suisse française (1855), Eusèbe-Henri Gaullieur est le premier à dresser une liste, non exhaustive, des pièces «indigènes" 5 . Le répertoire romand brille cependant par son extrême indigence, car cette liste comprend essentiellement des pièces de Français de passage sur sol suisse, à l'exemple de Joseph Patrat, Joseph-François Gallier de Saint-Gérand et Louis-Sébastien Mercier, ainsi que celles d'un officier saint-gallois, Muller de Friedberg, imprimées à Neuchâtel. Les pièces de ce dernier «sont assez bien conçues, dans le genre du drame allemand; mais encore une fois, on voit en les lisant que la veine dramatique n'est pas précisément la nôtre» ${ }^{6}$, conclut Gaullieur.

Ce n'est donc pas le fruit du hasard si l'historiographie du $\mathrm{XX}^{\mathrm{e}}$ siècle s'est essentiellement focalisée sur l'émergence et le succès à l'échelle nationale d'un nouveau type de spectacle: les grandes pièces historiques à caractère patriotique, écrites, mises en scène et en musique, et interprétées par de réels «indigènes", dont le Festspiel est l'incarnation la plus emblématique. À partir de la fin du XIX ${ }^{\mathrm{e}}$ siècle, le théâtre romand possède enfin des dramaturges, un répertoire qui s'étoffe progressivement, des scènes dignes de ce nom, bref une histoire. Mais cette histoire du théâtre romand reste à ce jour jalonnée de lacunes, parfois béantes.

L'idée d'organiser un colloque portant sur le théâtre dramatique et lyrique en Suisse romande a germé en 2016 déjà : il semblait important pour Olivier Robert et moi-même, qui travaillions chacun depuis une dizaine d'années sur le théâtre vaudois, de faire le point sur les connaissances actuelles et surtout de stimuler l'intérêt autour de cet objet d'étude quelque peu délaissé par la recherche ${ }^{7}$. Organisé les 5 et 6 octobre 2018 au théâtre de La Grange à Dorigny, ce colloque avait pour double objectif d'explorer la vie théâtrale romande à une époque où ce divertissement n'est pas encore institutionnalisé, depuis le milieu du XVIII e siècle - période où l'on assiste à un essor des arts du spectacle - jusqu'à l'entre-deux-guerres, ainsi que de favoriser le dialogue

5. E.-H. Gaullieur, Études sur l'histoire littéraire de la Suisse française, p. 240-247.

6. Ibid., p. 247.

7. Ces trente dernières années, rares sont les chercheurs à s'être penchés sur le sujet pour la période précédant la Seconde Guerre mondiale: Ariane Girard (pour le théâtre genevois), Yvonne Tissot (théâtre neuchâtelois), Hubertus von Gemmingen (théâtre jésuite fribourgeois) et Joël Aguet, responsable entre autres de la partie romande du Dictionnaire du théâtre en Suisse (2005). 
entre historiens, littéraires, musicologues et historiens de l'art, souvent cloisonnés dans leur champ d'étude.

Le présent volume est le résultat d'une rencontre très fructueuse entre des chercheurs provenant de diverses disciplines et qui sont, pour la grande majorité d'entre eux, non spécialistes de la question. Il met en lumière les enjeux socioculturels du théâtre en Suisse romande sur une période de deux cents ans environ. Au fil de la lecture des quatorze contributions se dessine peu à peu une fresque mettant en valeur les différentes pratiques et fonctions sociales du théâtre, ses enjeux symboliques et identitaires. Notre ouvrage ne prétend pas avoir épuisé le sujet, bien au contraire $^{8}$; nous souhaitons avant tout démontrer l'intérêt de s'y pencher davantage et de mettre au jour de nouvelles archives, de nouveaux sujets. Nombreux sont les fonds encore inexploités ou qui méritent d'être étudiés avec une approche nouvelle, la plus interdisciplinaire possible.

Composé de trois parties distinctes, l'ouvrage s'intéresse en premier lieu à l'essor du théâtre dans la région lémanique et à son appropriation parfois tumultueuse. Les contributions de Marco Cicchini et Lia Leveillé Mettral explorent les enjeux liés au théâtre public à Genève, que ce soit sous l'angle de la police ou celui du comité des actionnaires et des directeurs du Théâtre des Bastions. Les apports de Jennifer Ruimi, Béatrice Lovis et Aline Hodroge mettent, quant à eux, en valeur le succès sans précédent du théâtre de société auprès des élites dans la seconde moitié du XVIII ${ }^{\mathrm{e}}$ siècle et au début du XIX ${ }^{\mathrm{e}}$ siècle, tout en soulignant l'importance en terres genevoises et vaudoises de quelques figures marquantes que sont Voltaire, Isabelle de Montolieu et Germaine de Staël. À la fois organisatrices, actrices et dramaturges, ces dernières ont aussi pour point commun d'avoir renoncé à publier leurs propres pièces, qui paraîtront de manière posthume ou resteront à l'état de manuscrit, un sort fréquent pour les pièces créées en société devant un parterre d'amis et, qui plus est, écrites par des plumes féminines.

La deuxième partie évoque l'intérêt grandissant des Romands pour le théâtre amateur qui se démocratise dans le courant du XIX siècle. Celui-ci se manifeste d'une part dans un cadre privé relativement confidentiel, proche du modèle de l'Ancien Régime, à l'exemple des «folies» volontiers burlesques rédigées par Rodolphe Töpffer pour les

8. Nous regrettons notamment l'absence de contributions portant sur les cantons catholiques (Jura, Fribourg et Valais). 
élèves de son pensionnat (Daniel Maggetti) ou des pièces beaucoup plus sages de Juste Olivier, qui oscillent entre un théâtre d'éducation "pour demoiselles» et un théâtre de lecture situé dans un décor exaltant la beauté des paysages suisses et des valeurs morales très helvétiques (Valentina Ponzetto). D’autre part, dès la seconde moitié du siècle, on assiste à une multiplication des spectacles publics proposés aussi bien par des sociétés d'étudiants, avec les théâtrales de Zofingue, Belles-Lettres et Helvetia (Alain Corbellari), que par des troupes amateurs, dont La Muse est l'une des plus actives (Olivier Robert). Celle-ci jouera un rôle primordial pour la création d'un théâtre romand en mettant en scène des auteurs locaux comme René Morax, Henri Warnéry ou Virgile Rossel.

Le dernier volet est consacré aux affirmations identitaires sur lesquelles se construit le théâtre romand dès la fin du XIX ${ }^{e}$ siècle. L'identité nationale est régulièrement mise en spectacle, comme le rappellent Philippe Kaenel et Danielle Chaperon, qui ont proposé à des étudiants en Master de se pencher sur cette thématique lors de leurs travaux de séminaire, des travaux dont les résultats sont désormais en ligne sur le site de l'Université de Lausanne. Le subside du Conseil fédéral alloué à la construction du Théâtre du Jorat et la présence régulière des conseillers fédéraux aux représentations méziéroises, privilège dont seul le Jorat a bénéficié, démontrent la volonté des autorités politiques de favoriser l'émergence d'un théâtre suisse. Cependant, le rêve de René Morax de créer dans la campagne de Mézières un théâtre par et pour le peuple, dans le sillage de l'utopie du Théâtre du peuple à Bussang, se confrontera vite à la réalité d'un contexte sociopolitique tendu, marqué par la montée des sentiments patriotiques et nationalistes, comme le démontre Léo Biétry. Le répertoire du Théâtre du Jorat laisse la part belle à la musique qui concourt, elle aussi, à donner une couleur helvétique aux spectacles: avec Henriette, qui inaugure la "Grange Sublime» en 1908, Gustave Doret ponctue la partition de solutions musicales pour la rendre abordable aux chœurs amateurs et afin que ses paroles soient intelligibles (Delphine Vincent). Si René Morax rencontre un large succès en terre romande avec ses pièces, cela ne sera pas le cas d'autres compatriotes qui tenteront de se frotter à la scène parisienne. Les projets d'Édouard Rod, romancier et critique renommé, se heurteront aux fortes contraintes régissant le monde théâtral de la capitale française et à des modèles esthétiques très éloignés des aspirations de l'auteur vaudois (Selina Follonier). Enfin, la contribution de Marc Perrenoud décortique les enjeux identitaires qui 
touchent, pendant l'entre-deux-guerres, les spectacles donnés dans le milieu ouvrier et la population juive de La Chaux-de-Fonds, cité horlogère dont la vie théâtrale s'épanouit depuis la construction en 1837 d'un très beau théâtre, récemment restauré.

Avant de conclure, les éditeurs de cet ouvrage tiennent à remercier chaleureusement le comité scientifique qui les a conseillés et aidés pour la bonne tenue du colloque, à savoir François Rosset et Valentina Ponzetto, de la section de Français de l'Université de Lausanne, François Vallotton de la section d'Histoire, ainsi que Delphine Vincent, musicologue à l'Université de Fribourg. Ils remercient également les experts, cités nommément en début de volume, pour leurs lectures attentives des différentes contributions. Que les participants à la table ronde qui a clos le colloque, "Arts vivants dans des murs anciens: les défis à relever pour les théâtres romands fondés avant 1939», soient ici remerciés: Michel Caspari (dir. Théâtre du Jorat, Mézières), François Marin (dir. Théâtre de Valère, Sion), John Voisard (dir. TPR, La Chaux-de-Fonds) et JeanPierre Pastori, journaliste et modérateur de cette table ronde. Enfin, leur gratitude va à Dominique Hauser et à son équipe pour avoir accueilli l'événement à La Grange de Dorigny et permis de monter un spectacle de René Morax, Le Choix d'une déesse, qui a magnifiquement ponctué ces deux journées consacrées au théâtre en Suisse romande.

Béatrice Lovis Université de Lausanne 


\section{BIBLIOGRAPHIE}

Gaullieur, Eusèbe-Henri, Études sur l'histoire littéraire de la Suisse française, particulièrement dans la seconde moitié du XVIIIe siècle, coll. Bulletin de l'Institut national genevois 9, 1855.

Gindroz, André, Histoire de l'instruction publique dans le Pays de Vaud, Lausanne, Georges Bridel, 1853.

Maggetti, Daniel, L'invention de la littérature romande, 1830-1910, Lausanne, Payot, 1995.

Töpffer, Rodolphe, Voyage autour du Mont-Blanc [1843], in Nouveaux voyages en zigzag, avec une notice de Sainte-Beuve, Paris, Victor Lecou, 1859. 
